\title{
A Finite Difference Method of High Order Accuracy for the Solution of Two-Point Boundary Value Problems
}

\author{
P.K. Pandey \\ Department of Mathematics \\ Dyal Singh College (University of Delhi) \\ Lodhi Road, New Delhi - 110003, India \\ E-mail: pramod_10p@hotmail.com
}

Abstract: We present a new high order finite difference method for second order differential equation $\mathrm{y}^{\prime \prime}(\mathrm{x})=\mathrm{f}(\mathrm{x}, \mathrm{y})$ subject to boundary conditions $y(a)=\alpha$ and $y(b)=\beta$. The method is based on rational function approximation and its development is based on power series expansions. Under appropriate conditions, local truncation error calculated and order of method estimated six. Our finite difference method leads to nonlinear system of equations. Numerical examples are given to illustrate the effectiveness, efficiency and high order accuracy of the method.

Keywords: differential equations, sixth order method, finite difference method, rational approximations, Troesch's test problem.

\section{Introduction}

In this paper, we consider numerical solution of the boundary value problem

$$
\mathrm{y}^{\prime \prime}(\mathrm{x})=\mathrm{f}(\mathrm{x}, \mathrm{y}), \quad \mathrm{x} \in[\mathrm{a}, \mathrm{b}] \subset \mathbb{R}, \quad \mathrm{f}(\mathrm{x}, \mathrm{y}), \mathrm{y}(\mathrm{x}) \in \mathbb{R}
$$

subject to boundary conditions $\mathrm{y}(\mathrm{a})=\alpha$, and $\mathrm{y}(\mathrm{b})=\beta$, where $\alpha, \beta$ are real finite constants.

The existence and uniqueness of the solution for the problem (1) is assumed. The specific assumption on $\mathrm{f}(\mathrm{x}, \mathrm{y})$, to ensure existence and uniqueness will not be considered $[1,2]$.

A second and fourth order three point discretization for problem (1) is well known [2, $3,4]$. A sixth order three point discretization for problem (1) is given by Chawla [5]. These methods based on the polynomial functions, which are normally smooth and sufficient continuous derivatives. These methods are used to solve specific differential equation. 
In the present paper, we describe a sixth order finite difference method for differential equation (1). The method is based on rational function approximation and its development based on power series expansions (Taylor and binomial). Our difference method leads to a nonlinear system of equations. A significant feature of our method is that each discretization of the differential equation at an interior grid point is based on three evaluations of $f(x, y)$. In section 2 , we discuss the derivation and development of the method. In section 3, under appropriate conditions obtain the local truncation error. In section 4, we consider numerical examples to illustrate the effectiveness and efficiency of the method. We also compare the performance of the present method with the existing sixth order method given by Chawla in [5].

\section{Derivation and Development of the method}

An idea of rational function approximation method for solution of two-point boundary value problems and development can be found in the literature $[6,7]$.

Let $\mathrm{N}$ be positive integer, $\mathrm{h}=(\mathrm{b}-\mathrm{a}) / \mathrm{N}$ and $\mathrm{x}_{\mathrm{i}}=\mathrm{a}+\mathrm{i} . \mathrm{h}, \mathrm{i}=0$ (1) N. The value of exact solution $y(x)$ at grid point $x_{i}$ are denoted by $y_{i}$, similarly $y_{i}^{\prime \prime}=f\left(x_{i}, y_{i}\right)=f_{i}$ etc.

Suppose we have to determine a number $y_{i}$, which is an approximation to the value of the theoretical solution $y(x)$ of the problem (1) at the nodal point $x_{i}, i=0$ (1) $N$. Assuming local assumption that no previous truncation errors have been made [8] i.e. $y_{i-1}=y\left(x_{i-1}\right)$. For obtaining an approximate value $y_{i}$, of the theoretical solution $y\left(x_{i}\right)$ of the problem (1), that purpose, following the ideas in $[9,10]$, we propose multistep difference method as,

$$
y_{i+1}-2 y_{i}+y_{i-1}=\frac{\left.h^{2}\left(a_{3}\left(y_{i+1}^{\prime \prime}+y_{i-1}^{\prime \prime}\right)+a_{4} y_{i}^{\prime \prime}\right)\right)}{G\left(x_{i}+h\right)+a_{0} y_{i+1}+a_{1} y_{i-1}+a_{2} y_{i}}
$$

where $\mathrm{G}(\mathrm{x})$, is an unknown sufficiently differentiable function of step length $\mathrm{h}$ and coefficients $a_{i}, i=0$ (1) 4 , are unknown constants to be determined under appropriate restrictions and conditions. Also we assumed that

$$
\mathrm{G}\left(\mathrm{x}_{\mathrm{i}}+\mathrm{h}\right)+\mathrm{a}_{0} \mathrm{y}_{\mathrm{i}+1}+\mathrm{a}_{1} \mathrm{y}_{\mathrm{i}-1}+\mathrm{a}_{2} \mathrm{y}_{\mathrm{i}} \neq 0 \text { for any } \mathrm{i}=1 \text { (1) } \mathrm{N}-1
$$

Let define a function $\mathrm{F}_{\mathrm{i}}(\mathrm{a}, \mathrm{h}, \mathrm{y})$ and associate (2) with it as,

$$
\begin{gathered}
\mathrm{F}_{\mathrm{i}}(\mathrm{a}, \mathrm{h}, \mathrm{y})=\left(\mathrm{y}\left(\mathrm{x}_{\mathrm{i}}+\mathrm{h}\right)-2 \mathrm{y}\left(\mathrm{x}_{\mathrm{i}}\right)+\mathrm{y}\left(\mathrm{x}_{\mathrm{i}}-\mathrm{h}\right)\right) \times\left(\mathrm{G}\left(\mathrm{x}_{\mathrm{i}}+\mathrm{h}\right)+\mathrm{a}_{0} \mathrm{y}\left(\mathrm{x}_{\mathrm{i}}+\mathrm{h}\right)+\right. \\
\left.\mathrm{a}_{1} \mathrm{y}\left(\mathrm{x}_{\mathrm{i}}-\mathrm{h}\right)+\mathrm{a}_{2} \mathrm{y}\left(\mathrm{x}_{\mathrm{i}}\right)\right)-\mathrm{h}^{2}\left(\mathrm{a}_{3}\left(\mathrm{y}^{\prime \prime}\left(\mathrm{x}_{\mathrm{i}}+\mathrm{h}\right)+\mathrm{y}^{\prime \prime}\left(\mathrm{x}_{\mathrm{i}}-\mathrm{h}\right)\right)+\mathrm{a}_{4} \mathrm{y}^{\prime \prime}\left(\mathrm{x}_{\mathrm{i}}\right)\right)=0
\end{gathered}
$$

Let us expand $G\left(x_{i}+h\right)$, in Taylor series about point $x=x_{i}$,

$G(h)=G\left(x_{i}\right)+h G^{\prime}\left(x_{i}\right)+\frac{h^{2}}{2 !} G^{\prime \prime}\left(x_{i}\right)+\frac{h^{3}}{3 !} G^{(3)}\left(x_{i}\right)+\frac{h^{4}}{4 !} G^{(4)}\left(x_{i}\right)+\frac{h^{5}}{5 !} G^{(5)}\left(x_{i}\right)+O\left(h^{6}\right)$

where $G^{\prime}$ denotes first derivative of $G(h)$ w.r.t. $h, G^{\prime \prime}$.... etc.

Expand $\mathrm{F}_{\mathrm{i}}(\mathrm{a}, \mathrm{h}, \mathrm{y})$ in Taylor series about point $\mathrm{x}_{\mathrm{i}}$, we have 


$$
\begin{gathered}
\left(h^{2} y_{i}^{\prime \prime}+\frac{h^{4}}{12} y_{i}^{(4)}+\frac{h^{6}}{360} y_{i}^{(6)}+O\left(h^{8}\right)\right) \times\left(G\left(x_{i}+h\right)+\left(a_{0}+a_{1}+a_{2}\right) y_{i}+\right. \\
h\left(a_{0}-a_{1}\right) y_{i}^{\prime}+\frac{h^{2}}{2 !}\left(a_{0}+a_{1}\right) y_{i}^{\prime \prime}+\frac{h^{3}}{3 !}\left(a_{0}-a_{1}\right) y_{i}^{(3)}+\frac{h^{4}}{4 !}\left(a_{0}+a_{1}\right) y_{i}^{(4)}+\frac{h^{5}}{5 !}\left(a_{0}-\right. \\
\left.\left.a_{1}\right) y_{i}^{(5)}+O\left(h^{6}\right)\right)-h^{2}\left(\left(2 a_{3}+a_{4}\right)+a_{3} h^{2} y_{i}^{(4)}+a_{3} \frac{h^{4}}{12} y_{i}^{(6)}\right)=0
\end{gathered}
$$

Using (4) in (5) and rewrite it as,

$$
\begin{gathered}
\mathrm{h}^{2}\left(\mathrm{~A}_{0}-2 \mathrm{a}_{3}-\mathrm{a}_{4}\right) \mathrm{y}_{\mathrm{i}}^{\prime \prime}+\mathrm{h}^{3} \mathrm{~A}_{1} \mathrm{y}_{\mathrm{i}}^{\prime \prime}+\frac{\mathrm{h}^{4}}{12}\left(\left(\mathrm{~A}_{0}-12 \mathrm{a}_{3}\right) \mathrm{y}_{\mathrm{i}}^{(4)}+6 \mathrm{~A}_{2} \mathrm{y}_{\mathrm{i}}^{\prime \prime}\right)+\frac{\mathrm{h}^{5}}{12}\left(\mathrm{~A}_{1} \mathrm{y}_{\mathrm{i}}^{(4)}+\right. \\
\left.2 \mathrm{~A}_{3} \mathrm{y}_{\mathrm{i}}^{\prime \prime}\right)+\frac{\mathrm{h}^{6}}{360}\left(\left(\mathrm{~A}_{0}-30 \mathrm{a}_{3}\right) \mathrm{y}_{\mathrm{i}}^{(6)}+15 \mathrm{~A}_{2} \mathrm{y}_{\mathrm{i}}^{(4)}+15 \mathrm{~A}_{4} \mathrm{y}_{\mathrm{i}}^{\prime \prime}\right)+\frac{\mathrm{h}^{7}}{360}\left(5 \mathrm{~A}_{3} \mathrm{y}_{\mathrm{i}}^{(4)}+3 \mathrm{~A}_{5} \mathrm{y}_{\mathrm{i}}^{\prime \prime}\right)+ \\
\mathrm{O}\left(\mathrm{h}^{8}\right)=0
\end{gathered}
$$

where

$$
\begin{gathered}
\mathrm{A}_{0}=\mathrm{G}\left(\mathrm{x}_{\mathrm{i}}\right)+\left(\mathrm{a}_{0}+\mathrm{a}_{1}+\mathrm{a}_{2}\right) \mathrm{y}_{\mathrm{i}} \\
\mathrm{A}_{\mathrm{k}}=\mathrm{G}^{(\mathrm{k})}\left(\mathrm{x}_{\mathrm{i}}\right)+\left(\mathrm{a}_{0}+(-1)^{\mathrm{k}} \mathrm{a}_{1}\right) \mathrm{y}_{\mathrm{i}}^{(\mathrm{k})} \quad, \mathrm{k}=1(1) 5
\end{gathered}
$$

If we equate the coefficient of $h^{j}, j=2(1) 7$ in (6) both side, we will obtained

$$
\begin{gathered}
\left(\mathrm{A}_{0}-2 \mathrm{a}_{3}-\mathrm{a}_{4}\right) \mathrm{y}_{\mathrm{i}}^{\prime \prime}=0 \\
\mathrm{~A}_{1} \mathrm{y}_{\mathrm{i}}^{\prime \prime}=0 \\
\left(\mathrm{~A}_{0}-12 \mathrm{a}_{3}\right) \mathrm{y}_{\mathrm{i}}^{(4)}+6 \mathrm{~A}_{2} \mathrm{y}_{\mathrm{i}}^{\prime \prime}=0 \\
\mathrm{~A}_{1} \mathrm{y}_{\mathrm{i}}^{(4)}+2 \mathrm{~A}_{3} \mathrm{y}_{\mathrm{i}}^{\prime \prime}=0 \\
\left(\mathrm{~A}_{0}-30 \mathrm{a}_{3}\right) \mathrm{y}_{\mathrm{i}}^{(6)}+15 \mathrm{~A}_{2} \mathrm{y}_{\mathrm{i}}^{(4)}+15 \mathrm{~A}_{4} \mathrm{y}_{\mathrm{i}}^{\prime \prime}=0 \\
5 \mathrm{~A}_{3} \mathrm{y}_{\mathrm{i}}^{(4)}+3 \mathrm{~A}_{5} \mathrm{y}_{\mathrm{i}}^{\prime \prime}=0
\end{gathered}
$$

If we assume that $y_{i}^{(p)} \neq 0,=1(1) 6$, and

$$
\mathrm{A}_{0}-30 \mathrm{a}_{3}=0
$$

Thus we obtained,

$$
\begin{gathered}
\mathrm{A}_{0}-2 \mathrm{a}_{3}-\mathrm{a}_{4}=0 \\
\mathrm{~A}_{1}=\mathrm{A}_{3}=\mathrm{A}_{5}=0 \\
\left(\mathrm{~A}_{0}-12 \mathrm{a}_{3}\right) \mathrm{y}_{\mathrm{i}}^{(4)}+6 \mathrm{~A}_{2} \mathrm{y}_{\mathrm{i}}^{\prime \prime}=0 \\
5 \mathrm{~A}_{3} \mathrm{y}_{\mathrm{i}}^{(4)}+3 \mathrm{~A}_{5} \mathrm{y}_{\mathrm{i}}^{\prime \prime}=0
\end{gathered}
$$

Solving system of equations (7-8), we obtained

$$
\begin{gathered}
\mathrm{a}_{4}=28 \mathrm{a}_{3} \\
\mathrm{G}\left(\mathrm{x}_{\mathrm{i}}\right)=30 \mathrm{a}_{3}-\left(2 \mathrm{a}_{0}+\mathrm{a}_{2}\right) \mathrm{y}_{\mathrm{i}}
\end{gathered}
$$




$$
\begin{gathered}
G^{(2 m)}\left(x_{i}\right)=-2 a_{0} y_{i}^{(2 m)}+3 a_{3}\left(-\frac{y_{i}^{(4)}}{y_{i}^{\prime \prime}}\right)^{m}, m=1,2 \\
G^{\prime}\left(x_{i}\right)=G^{(3)}\left(x_{i}\right)=G^{(5)}\left(x_{i}\right)=0
\end{gathered}
$$

with the help of (4) and (9), from (2), after neglecting $\mathrm{O}\left(\mathrm{h}^{6}\right)$, we find that

$$
y_{i+1}-2 y_{i}+y_{i-1}=\frac{8 h^{2}\left(y_{i+1}^{\prime \prime}+28 y_{i}^{\prime \prime}+y_{i-1}^{\prime \prime}\right)\left(y_{i}^{\prime \prime}\right)^{2}}{240\left(y_{i}^{\prime \prime}\right)^{2}-12 h^{2} y_{i}^{\prime \prime} y_{i}^{(4)}+h^{4}\left(y_{i}^{(4)}\right)^{2}}
$$

If we replace $y_{i}^{(4)}$ in (10) by second order difference formulae

we have

$$
\frac{\left(y_{i+1}^{\prime \prime}-2 y_{i}^{\prime \prime}+y_{i-1}^{\prime \prime}\right)}{h^{2}}
$$

$$
y_{i+1}-2 y_{i}+y_{i-1}=\frac{8 h^{2}\left(y_{i+1}^{\prime \prime}+28 y_{i}^{\prime \prime}+y_{i-1}^{\prime \prime}\right)\left(y_{i}^{\prime \prime}\right)^{2}}{240\left(y_{i}^{\prime \prime}\right)^{2}-12 y_{i}^{\prime \prime}\left(y_{i+1}^{\prime \prime}-2 y_{i}^{\prime \prime}+y_{i-1}^{\prime \prime}\right)+\left(y_{i+1}^{\prime \prime}-2 y_{i}^{\prime \prime}+y_{i-1}^{\prime \prime}\right)^{2}}
$$

Using a notation as defined above $\mathrm{y}_{\mathrm{i}}^{\prime \prime}=\mathrm{f}\left(\mathrm{x}_{\mathrm{i}}, \mathrm{y}_{\mathrm{i}}\right)=\mathrm{f}_{\mathrm{i}}$, from (11) we get our rational approximation method for numerical solution of problem (1) as

$$
y_{i+1}-2 y_{i}+y_{i-1}=\frac{8 h^{2}\left(f_{i+1}+28 f_{i}+f_{i-1}\right)\left(f_{i}\right)^{2}}{240\left(f_{i}\right)^{2}-12 f_{i}\left(f_{i+1}-2 f_{i}+f_{i-1}\right)+\left(f_{i+1}-2 f_{i}+f_{i-1}\right)^{2}}
$$

A non-linear multistep method is usually designed for dealing with unconventional problems such as initial-value problem, problem with singularities for which classical methods generally performs poorly. It was noticed that some of the non-linear multistep method which designed for singular problems do not perform on non- singular problems [11]. The proposed method (10), a nonlinear multistep method designed to solve unspecific problem (1). For each nodal point, we will obtain the nonlinear system of equation given by [12] or a linear system of equations if the source function is $f(x)$. Thus the obtained resulting system of nonlinear equations (12) is solved by NewtonRaphson method in considered model problems. In numerical section we will see that the proposed method is suitable for both linear and nonlinear problem. The computational results show that proposed method is effective and accurate.

\section{Local Truncation Error}

It follows from construction of the method (10) that the new method is at least of six orders. In fact if we consider the local truncation error as defined in [12,13], of the proposed difference method (10), may be written as

$$
\mathrm{T}_{\mathrm{i}}=\mathrm{y}_{\mathrm{i}+1}-2 \mathrm{y}_{\mathrm{i}}+\mathrm{y}_{\mathrm{i}-1}-\frac{\mathrm{h}^{2}}{30}\left(\mathrm{y}_{\mathrm{i}+1}^{\prime \prime}+28 \mathrm{y}_{\mathrm{i}}^{\prime \prime}+\mathrm{y}_{\mathrm{i}-1}^{\prime \prime}\right) \times\left(1-\frac{\mathrm{h}^{2}}{20}\left(\frac{\mathrm{y}_{\mathrm{i}}^{(4)}}{\mathrm{y}_{\mathrm{i}}^{\prime \prime}}\right)+\frac{\mathrm{h}^{4}}{240}\left(\frac{\mathrm{y}_{\mathrm{i}}^{(4)}}{\mathrm{y}_{\mathrm{i}}^{\prime \prime}}\right)^{2}\right)^{-1}
$$

Write Taylor series expansion for each term in (13) about point $\mathrm{x}_{\mathrm{i}}$, we have 


$$
\begin{gathered}
\left(\mathrm{h}^{2} \mathrm{y}_{\mathrm{i}}^{\prime \prime}+\frac{\mathrm{h}^{4}}{12} \mathrm{y}_{\mathrm{i}}^{(4)}+\frac{\mathrm{h}^{6}}{360} \mathrm{y}_{\mathrm{i}}^{(6)}+\mathrm{O}\left(\mathrm{h}^{8}\right)\right)-\frac{\mathrm{h}^{2}}{30}\left(30 \mathrm{y}_{\mathrm{i}}^{\prime \prime}+\mathrm{h}^{2} \mathrm{y}_{\mathrm{i}}^{(4)}+\frac{\mathrm{h}^{4}}{12} \mathrm{y}_{\mathrm{i}}^{(6)}\right) \times(1- \\
\left.\frac{\mathrm{h}^{2}}{20}\left(\frac{\mathrm{y}_{\mathrm{i}}^{(4)}}{\mathrm{y}_{\mathrm{i}}^{\prime \prime}}\right)+\frac{\mathrm{h}^{4}}{240}\left(\frac{\mathrm{y}_{\mathrm{i}}^{(4)}}{\mathrm{y}_{\mathrm{i}}^{\prime \prime}}\right)^{2}\right)^{-1}
\end{gathered}
$$

Assume $\mathrm{h}^{4}\left(\mathrm{y}_{\mathrm{i}}^{(4)}\right)^{2}-240\left(\mathrm{y}_{\mathrm{i}}^{\prime \prime}\right)^{2}<\mathrm{h}^{2} \mathrm{y}_{\mathrm{i}}^{\prime \prime} \mathrm{y}_{\mathrm{i}}^{(4)}<\mathrm{h}^{4}\left(\mathrm{y}_{\mathrm{i}}^{(4)}\right)^{2}+240\left(\mathrm{y}_{\mathrm{i}}^{\prime \prime}\right)^{2}$, then by application of binomial theorem on right side of (14), we have

$$
\begin{gathered}
\left(\mathrm{h}^{2} \mathrm{y}_{\mathrm{i}}^{\prime \prime}+\frac{\mathrm{h}^{4}}{12} \mathrm{y}_{\mathrm{i}}^{(4)}+\frac{\mathrm{h}^{6}}{360} \mathrm{y}_{\mathrm{i}}^{(6)}+\mathrm{O}\left(\mathrm{h}^{8}\right)\right)-\frac{\mathrm{h}^{2}}{30}\left(30 \mathrm{y}_{\mathrm{i}}^{\prime \prime}+\mathrm{h}^{2} \mathrm{y}_{\mathrm{i}}^{(4)}+\frac{\mathrm{h}^{4}}{12} \mathrm{y}_{\mathrm{i}}^{(6)}\right) \times\left(1+\frac{\mathrm{h}^{2}}{20} \frac{\mathrm{y}_{\mathrm{i}}^{(4)}}{\mathrm{y}_{\mathrm{i}}^{\prime \prime}}-\right. \\
\left.\frac{\mathrm{h}^{4}}{600}\left(\frac{\mathrm{y}_{\mathrm{i}}^{(4)}}{\mathrm{y}_{\mathrm{i}}^{\prime \prime}}\right)^{2}+\mathrm{O}\left(\mathrm{h}^{6}\right)\right)=\left(\mathrm{h}^{2} \mathrm{y}_{\mathrm{i}}^{\prime \prime}+\frac{\mathrm{h}^{4}}{12} \mathrm{y}_{\mathrm{i}}^{(4)}+\frac{\mathrm{h}^{6}}{360} \mathrm{y}_{\mathrm{i}}^{(6)}+\mathrm{O}\left(\mathrm{h}^{8}\right)\right)-\frac{\mathrm{h}^{2}}{30}\left(30 \mathrm{y}_{\mathrm{i}}^{\prime \prime}+\frac{5 \mathrm{~h}^{2}}{2} \mathrm{y}_{\mathrm{i}}^{(4)}+\right. \\
\left.\frac{\mathrm{h}^{4}}{12} \mathrm{y}_{\mathrm{i}}^{(6)}+\mathrm{O}\left(\mathrm{h}^{6}\right)\right)=\mathrm{O}\left(\mathrm{h}^{8}\right)
\end{gathered}
$$

Thus from (15), we conclude that proposed method (10) has at least six order accuracy.

\section{Numerical Experiments}

In this section, the proposed method (12) is applied to solve four different model problems. The resulting system of nonlinear equations so obtained solved by NewtonRaphson method. All computations were performed in GNU FORTRAN language, using double precision. Let $y_{i}$, the numerical value calculated by formulae (12), an approximate value of the theoretical solution $y(x)$ at the grid pointx $=x_{i}$. The maximum absolute error

$$
\operatorname{MAE}(y)=\max _{1 \leq \mathrm{i} \leq \mathrm{N}-1}\left|\mathrm{y}\left(\mathrm{x}_{\mathrm{i}}\right)-\mathrm{y}_{\mathrm{i}}\right|
$$

are shown in Tables 1-4, for different value of $h$, the step length. Also we have computed $y_{i}$, using formula given by Chawla [5] and shown in the tables.

Example 1. Consider the following two point boundary value problem

$$
\mathrm{y}^{\prime \prime}(\mathrm{x})=\frac{2}{\mathrm{x}^{2}} \mathrm{y}(\mathrm{x})-\frac{1}{\mathrm{x}}, \quad 1<\mathrm{x}<2
$$

with the boundary conditions $y(1)=\frac{11}{19}, y(2)=0$. In Table 1 , the maximum absolute error presented in exact solution $(x)=\left(19 x-5 x^{2}-36 / x\right) / 38$.

Example 2. Consider the following nonlinear two-point boundary value problem

$$
\mathrm{y}^{\prime \prime}(\mathrm{x})=\frac{3}{2} \mathrm{y}^{2}(\mathrm{x}), \quad 0<\mathrm{x}<1
$$

with the boundary conditions $\mathrm{y}(0)=4, \mathrm{y}(1)=1$.In Table 2, the MAE presented in exact solution $\mathrm{y}(\mathrm{x})=4 /(1+\mathrm{x})^{2}$.

Example 3. Consider the following nonlinear two-point boundary value problem 


$$
\mathrm{y}^{\prime \prime}(\mathrm{x})=\frac{1}{2}(1+\mathrm{y}+\mathrm{x})^{3}, \quad 0<\mathrm{x}<1
$$

with the boundary conditions $y(0)=0=y(1)$. In Table 3 , the MAE presented in exact solution $\mathrm{y}(\mathrm{x})=-\mathrm{x}-1+2 /(2-\mathrm{x})$.

Example 4. Consider the Troesch's problem [14].

$$
\mathrm{y}^{\prime \prime}(\mathrm{x})=\alpha \operatorname{Sinh}(\alpha \mathrm{y})+\mathrm{f}(\mathrm{x}), \quad 0<\mathrm{x}<1
$$

with the boundary conditions $y(0)=0$ and $y(1)=\operatorname{Sinh}(1)$. In Table 4, the MAE presented in exact solution $\mathrm{y}(\mathrm{x})=\operatorname{Sinh}(\mathrm{x})$, for $\alpha=.5,1.0,2.0$.

Table 1. Maximum absolute error $y(x)=\left(19 x-5 x^{2}-36 / x\right) / 38$ in for example 1

\begin{tabular}{|c|c|c|c|c|c|}
\hline \multicolumn{2}{|c|}{ Error } & \multicolumn{4}{c|}{ Step length (h) } \\
\cline { 3 - 6 } & MAE & $.13332743(-3)$ & $.88431334(-5)$ & $.46868072(-6)$ & $.62557199(-7)$ \\
\hline \multirow{2}{*}{$(10)$} & & & & & \\
\hline Chawla's & MAE & $.17962958(-5)$ & $.26665235(-7)$ & $.28091184(-7)$ & $.67117142(-7)$ \\
\hline
\end{tabular}

Table 2. Maximum absolute error in $y(x)=4 /(1+x)^{2}$ for example 2

\begin{tabular}{|c|c|c|c|c|c|}
\hline \multicolumn{2}{|c|}{ Error } & \multicolumn{4}{c|}{ Step length (h) } \\
\cline { 3 - 6 } & MAE & $.14692306(-3)$ & $.65165545(-5)$ & $.24017024(-6)$ & $.47508550(-6)$ \\
\hline \multirow{2}{*}{$(10)$} & & & & & 64 \\
\hline Chawla's & MAE & $.13908767(-2)$ & $.33620209(-3)$ & $.70991518(-4)$ & $.28845704(-5)$ \\
\hline
\end{tabular}

Table 3. Maximum absolute error in $y(x)=-x-1+\frac{2}{2-x}$ for example 3

\begin{tabular}{|c|c|c|c|c|c|}
\hline \multicolumn{2}{|c|}{ Error } & \multicolumn{4}{c|}{ Step length (h) } \\
\cline { 3 - 6 }$(10)$ & MAE & $.24302900(-3)$ & $.16158820(-4)$ & $.76591971(-6)$ & $.16093255(-7)$ \\
\hline Chawla's & MAE & $.50297973(-3)$ & $.76657532(-4)$ & $.10819662(-4)$ & $.83681783(-6)$ \\
\hline
\end{tabular}

Table 4. Maximum absolute error in $y(x)=\operatorname{Sinh}(x)$ for example 4

\begin{tabular}{|c|c|c|c|c|c|}
\hline \multicolumn{2}{|c|}{ Error } & \multicolumn{2}{c|}{ Step length (h), $\alpha=.50$} & \multicolumn{2}{c|}{ Step length (h), $\alpha=1.0$} \\
\cline { 3 - 6 }$(10)$ & \multirow{2}{*}{ MAE } & 4 & 8 & 4 & 8 \\
\hline \multirow{2}{*}{ Chawla's } & $.14901161(-7)$ & $.29802322(-7)$ & $.95367432(-6)$ & $.59604645(-7)$ \\
& & & & & \\
\hline \multicolumn{2}{|c|}{ Error } & $.14901161(-7)$ & $.29802322(-7)$ & $.29802322(-7)$ & $.59604645(-7)$ \\
\cline { 3 - 6 } & & 4 & \multicolumn{3}{c|}{ Step length (h), $\alpha=2.0$} \\
\hline \multirow{2}{*}{$(10)$} & MAE & $.34451485(-4)$ & $.21457672(-5)$ & $.11920929(-6)$ & No change \\
\hline Chawla's & MAE & $.73480606(-3)$ & $.13351440(-4)$ & $.23841858(-6)$ & $.11920929(-6)$ \\
\hline
\end{tabular}




\section{Conclusion}

A new kind of finite difference scheme is presented for numerical solution of twopoint boundary value problems in ordinary differential equation. The local truncation error in the method (10) presented and is of order six. However numerical results only confirm that method has order more than four. The computational accuracy of present method can be improved if $\mathrm{y}_{\mathrm{i}}^{(4)}$ replaced by high order difference formulae. However it is satisfactory to obtain a comparable results using only three function evaluation unlike [5]. Our future work will deal with the presence of higher order derivative term in method to obtain the best computational result, the method can offer. Though method developed for solving two point boundary value problems in ODEs, it has good potential for efficient application to many problems in PDEs. The extension of present method to different problem is crucial. Work in this specific direction is in progress.

\section{References}

[1] Baxley, J.V.: Nonlinear Two Point Boundary Value Problems in Ordinary and Partial Differential Equations, Ordinary and Partial Differential Equations, Lecture Notes in Mathematics vol. 846, pp. 46-54, 1981

DOI: $10.1007 / \mathrm{BFb} 0089823$

[2] Keller, H.B.: Numerical Methods for Two Point Boundary Value Problems, Blaisdell Publishing Co., Waltham, Massachusetts, 1968

[3] Numerov, B.V.: A method of extrapolation of perturbations, Monthly Notices of the Royal Astronomical Society, vol. 84, no. 8, pp. 592-601, 1924

[4] Chawla, M.M.: A fourth order tridiagonal finite difference method for general nonlinear two point boundary value problems with mixed boundary conditions, Journal of the Institute of Mathematics and its Applications, vol. 20, no. 2, pp. 8393, 1977

[5] Chawla, M.M.: A sixth order tridiagonal finite difference method for nonlinear two point boundary value problems, BIT Numerical Mathematics, vol. 17, no. 2, pp. $128-133,1977$

DOI: $10.1007 / \mathrm{BF} 01932284$

[6] Mickens, R.E.: Nonstandard Finite Difference Models of Differential equations, World Scientific, Singapore, 1994

[7] Lambert, J.D.: Numerical Methods for Ordinary Differential Systems, John Wiley, Chichester, 1991

[8] Lambert, J.D., Shaw, B.: On the numerical solution of $y^{\prime}=f(x, y)$ by class of formulae based on rational approximation, Mathematics and Computation, vol. 19, no. 91, pp. 456-462, 1965

DOI: $10.1090 / \mathrm{S} 0025-5718-1965-0179947-7$

[9] Van Niekerk, F.D.: Non-linear one-step methods for initial value problems, Computers \& Mathematics with Applications, vol. 13, no. 4, pp. 367-371, 1987 DOI: $10.1016 / 0898-1221(87) 90004-6$

[10] Okosun, K.O., Ademiluyi, R.A.: A Three Step Rational Methods for Integration of Differential Equations with Singularities, Research Journal of Applied Sciences, vol. 2, no. 1, pp. 84-88, 2007

DOI: rjasci.2007.84.88 
[11] Abelman, S., Eyre, D.: A numerical Study of multistep methods based on continued fractions, Computers \& Mathematics with Applications, vol. 20, no. 8, pp. 51-60, 1990

DOI: 10.1016/0898-1221(90)90209-3

[12] Jain ,M.K.: Numerical Solution of Differential Equations (2nd ed.), Wiley Eastern Limited, New Delhi, 1984

[13] Stoer, J., Bulirsch, R.: Introduction to Numerical Analysis (2nd ed.), SpringerVerlag, Berlin Heidelberg, 1991

[14] Troesch, B.A.: A simple approach to a sensitive two-point boundary value problem, J. Comput. Phys, vol. 21, no. 3, pp. 279-290, 1976

DOI: $10.1016 / 0021-9991(76) 90025-5$ 